Service social

\title{
Expérience de coopération interuniversitaire en travail social : Hongrie-Québec
}

\author{
Denis Fortin, Lionel Groulx, André Jacob, Gisèle Legault, Justin Lévesque, \\ Robert Mayer et Étienne Vintze
}

Volume 37, numéro 1-2, 1988

Par-delà les barrières des sexes

URI : https://id.erudit.org/iderudit/706396ar

DOI : https://doi.org/10.7202/706396ar

Aller au sommaire du numéro

Éditeur(s)

École de service social de l'Université Laval

ISSN

1708-1734 (numérique)

Découvrir la revue

Citer ce document

Fortin, D., Groulx, L., Jacob, A., Legault, G., Lévesque, J., Mayer, R. \& Vintze, É. (1988). Expérience de coopération interuniversitaire en travail social :

Hongrie-Québec. Service social, 37(1-2), 253-268.

https://doi.org/10.7202/706396ar
Résumé de l'article

À partir d'un projet de coopération scientifique entre des professeurs et chercheurs québécois et leurs homologues hongrois, un colloque a eu lieu à Budapest du 2 au 6 juin 1987 et les participants en donnent un compte rendu et présentent une analyse de la situation du service social en Hongrie.

L'intérêt de l'expérience hongroise vient de l'omniprésence de l'État dans la sphère des activités de bien-être et du caractère avant-gardiste de certaines politiques familiales. 


\title{
Expérience de coopération interuniversitaire en travail social : Hongrie-Québec
}

\author{
Denis Fortin \\ Lionel Groulx \\ André Jacob \\ Gisèle Legault \\ Justin Lévesque* \\ Robert Mayer \\ Étienne Vintze
}

Le Regroupement des unités de formation universitaire en travail social (RUFUTS) du Québec s'intéresse depuis plusieurs années à la coopération internationale dans le domaine du service social. ${ }^{1}$

À l'été 1986, Ferge Zsuzsa, sociologue et professeure à l'Institut de sociologie de l'Académie des sciences de Hongrie (I.S.A.S.H.) et Étienne Vintze, travailleur social au Ministère de la justice du Québec, ont amorcé les premiers contacts avec André Jacob, alors responsable du comité des relations internationales du RUFUTS, afin de préparer un projet de coopération scientifique entre des professeurs et chercheurs québécois et leurs homologues hongrois. Ces contacts ont conduit à l'élaboration d'un protocole d'entente signé par Robert Mayer pour le RUFUTS et Ferge Zsuzsa pour l'I.S.A.S.H., qui a donné lieu à un colloque tenu à Budapest du 2 au 6 juin 1987.** Les participants québécois en donnent ici un compte rendu.

Un colloque de plusieurs jours avec des collègues de l'Europe de l'Est représentait une expérience unique de collaboration. Nous savons que la pratique du service social varie d'un contexte et d'un pays à l'autre ; l'expérience hongroise nous intéressait au plus haut point étant donné l'omniprésence de l'État dans la sphère des activités de bien-être et le caractère avant-gardiste de certaines politiques familiales. Bien sûr, notre trop court séjour ne nous a pas permis d'avoir des échanges avec des gens de tous les milieux. Par exemple, nous n'avons pas eu l'occasion de rencontrer des hauts fonctionnaires ou des dirigeants politiques afin de comparer leurs points de vue à celui de nos interlocuteurs. Malgré ces limites, nous tenterons de présenter une analyse relativement étoffée de la situation du service social en Hongrie, telle qu'elle nous fut présentée. 
Les principaux objectifs de l'entente visaient à promouvoir des échanges dans le domaine des politiques sociales, de l'enseignement, du développement de programmes de formation académique, de la recherche, des travaux scientifiques et des publications. Étaient également prévus des échanges sur les expériences de formation universitaire en travailsocial et sur l'implantation de ces divers programmes.

\section{Déroulement des activités du colloque}

À Budapest, nos entretiens se sont déroulés non loin de l'Académie des sciences, dans un centre de réunions qui lui est rattaché, et situé sur les collines de Buda, partie la plus élevée de la ville, dominant le Danube et offrant une vue exceptionnelle du fleuve.

Chaque rencontre regroupait une trentaine de personnes, majoritairement des praticiens, qui avaient été invitées par mesdames Ferge Zsuzsa et Lina Szilvasi (organisatrice locale du colloque) en raison de leur intérêt pour les thèmes abordés lors des séances de travail. Les trois premières journées ont été consacrées aux discussions académiques et au partage des expériences. Les deux autres journées ont été occupées à des visites d'organismes sociaux à Budapest et en province. Ces divers entretiens ont été quelque peu alourdis par des difficultés de communication en raison de l'unilinguisme des participants hongrois et de notre incapacité à communiquer dans leur langue ; la traduction a donc fait partie intégrante de nos échanges.

Nous avons d'abord fait le point sur la crise économique et son impact sur les politiques sociales. La réalité québécoise, sous l'angle de la double structure fédérale et provinciale en relation avec les politiques sociales, fut d'abord présentée. On souligna d'une façon particulière le contexte actuel : les coupures dans les services sociaux, le désengagement de l'État et le développement de "l'approche communautaire» pour suppléer à ce retrait progressif.

Les collègues hongrois ont également exposé la situation des politiques sociales dans leur pays. Alors qu'on avait longtemps présumé qu'une société socialiste résoudrait les problèmes de pauvreté, de chômage et d'inégalité en général, on a constaté, dans les années soixante, que cette structure économique avait engendré un certain nombre de problèmes sociaux et n'avait pas nécessairement réussi à les éviter tous. Alors que l'aide sociale avait été rattachée à l'emploi, la crise du plein d'emploi a fait émerger la notion de besoins sociaux en dehors du cadre strict du travail. Cette notion a à son tour été structurée autour de la famille, à laquelle l'économie socialiste s'est peu intéressée parce que perçue comme lieu de transmission de valeurs bourgeoises. $\AA$ 
partir des années soixante, les intervenants sociaux sont devenus des véhicules de l'autonomisation des familles, la société ne pouvant plus ou ne voulant plus suppléer aux fonctions de la famille. Les intervenants sociaux ont alors vécu ce dilemme, que nous connaissons également, d'assumer la double fonction de contrôle et de service auprès de la population.

La réforme de la santé et des services sociaux au Québec et le réseau actuel des Affaires sociales ont été présentés. Dans ce contexte, l'évolution des pratiques sociales québécoises et le rôle des établissements de services communautaires ont fait l'objet d'un thème particulier de discussion. En Hongrie, des centres d'aide à la famille ont été mis sur pied dans les années soixante-dix pour répondre aux besoins dans les secteurs enfance et personnes âgées. Dans ce pays où le service social n'existe pas comme profession autonome, les intervenants ne reçoivent donc pas de formation spécifique et ils sont, entre autres, enseignants, sociologues ou avocats. Reconnaître la nécessité de prodiguer des services sociaux les amène à penser à une formation mieux structurée. D'ailleurs, notre visite s'inscrivait dans cette perspective et coïncidait avec la première tentative de l'Académie des sciences, en contact avec le Ministère de la santé, de mettre sur pied un programme de formation professionnelle.

En ce qui concerne la formation en service social, nous avons présenté les particularités d'une formation de premier cycle. Les stages ont fait l'objet de discussions et l'importance de développer des habiletés, tant d'analyse que d'intervention, a été soulignée. Plusieurs autres sujets ont été discutés: la recherche sociale et, plus spécifiquement, recherche-action, politique familiale, divorce, médiation, situation des femmes, familles monoparentales, etc.

Nos partenaires hongrois ont ensuite retracé l'évolution des politiques familiales dans leur pays et ont rappelé l'attention accordée à la famille à partir des années soixante ; les politiques sociales, à ce chapitre, étant de fait des politiques de "population" ou démographiques, visant à encourager les naissances. Divers moyens incitatifs ont été mis sur pied à cet effet. On constate toutefois une inquiétude chez les praticiens hongrois en ce qui a trait aux soins aux enfants, les deux parents étant accaparés par le travail salarié, d'une part, et les ressources du couple étant souvent insuffisantes ou inadéquates, d'autre part. Nos interlocuteurs relient cette situation entre autres au cumul travail salarié/travail domestique des femmes et au cumul travail salarié/travail au noir des hommes.

Des rencontres avec des communautés spécifiques, à Budapest, au sud du pays et autour du Lac Balatton ont complété notre programme. 
Ainsi, une visite dans un foyer d'accueil pour jeunes filles intellectuellement déficientes nous a permis de constater le climat de chaleur et de confiance qui y régnait. Nos échanges avec les enfants ont porté essentiellement sur des questions ayant trait à la vie des jeunes québécois dans le contexte nord-américain. À travers les chants et les anecdotes folkloriques, nous avons appris à connaître un peu mieux la culture hongroise et à faire connaître la nôtre. Rencontres et repas communautaires avec les représentants de la communauté locale nous ont permis d'apprécier la cordialité et la chaleur du peuple hongrois.

Lors d'une visite dans une école primaire, nous avons également pu constater le dynamisme de la vie régionale et l'intérêt manifeste de l'université d'un grand centre comme Budapest, pour les réalités locales. À cette occasion, nous avons échangé sur les tendances nationales en éducation et en politiques sociales. Parallèlement, les gens se sont montrés intéressés par certains rapprochements avec les tendances nord-américaines et canadiennes dans ces mêmes secteurs.

\section{Conjoncture sociale, idéologique et politique}

Théoriquement, la relation socialisme/problèmes sociaux ne se présente pas tout à fait de la même manière que dans les pays capitalistes. Une approche traditionnelle et mécanique du socialisme comme système orienté vers la satisfaction des besoins sociaux, voulait que le " miracle" économique ou l'organisation socialiste de la production apporte toutes les solutions aux problèmes. En d'autres termes, on estimait que les surplus générés par la production devaient être appliqués automatiquement à la satisfaction des besoins sociaux. Or, avec les années, on a vite compris que les situations vécues par les gens ne trouvaient pas nécessairement leur explication et leur solution dans l'organisation logique, planifiée et déterministe des rapports de production.

Aujourd'hui, devant l'ampleur des problèmes psychosociaux et socio-économiques, cette conception mécaniciste de l'économie socialiste est sérieusement remise en cause. Comme le souligne Guzzetta, dans une analyse du développement du service social dans les pays socialistes, solutionner les problèmes de logement, d'emploi et de santé n'élimine pas pour autant les conflits conjugaux, la violence familiale, les problèmes de maladies mentales, l'alcoolisme et toutes les autres situations avec lesquelles sont aux prises les travailleurs sociaux. ${ }^{2}$ Plusieurs problèmes préoccupent autant les politiciens que la population.

Aujourd'hui, dans le contexte de la perestroïka à la mode hongroise, les dirigeants du pays reconnaissent un certain nombre d'erreurs et mettent tout en ouvre pour modifier la situation. D'ailleurs, le 20 mai 1988 à Budapest, a eu lieu une conférence nationale du Parti socialiste 
ouvrier hongrois qui a fait une très sérieuse autocritique des dernières années de gestion du gouvernement. On nous pardonnera une longue citation tirée d'une analyse de Temps nouveaux, mais elle nous semble utile pour illustrer le sérieux des réformes entreprises :

"[...] la multiplication des difficultés économiques et sociales a une incidence sur l'état d'esprit de la population. On voit s'exacerber le débat sur les voies de développement social, se manifester une confusion idéologique et s'affaiblir l'unité des communistes. [...] Le document dresse un constat réaliste de la situation. Il indique que durant les trois ans qui se sont écoulés depuis le congrès du P.S.O.H., la situation économique n'a pas connu d'amélioration. La dette extérieure en devises fortes a continué de s'accroître, l'inflation s'est accentuée, le fardeau des charges supportées par la population a augmenté. Le niveau de vie est en baisse, des tensions s'accumulent au sein de la société. Cela est dû en partie à des raisons objectives, liées au phénomène de crise que connaît l'économie mondiale, mais pour l'essentiel, souligne le projet de résolution, cela tient aux erreurs commises. " 3

Il est intéressant et réconfortant, en un sens, de voir les dirigeants du pays capables d'une telle autocritique, ce qui n'est pas toujours le cas chez nous.

En somme, on doit d'abord souligner que la crise économique internationale des dernières années a profondément affecté la Hongrie : la crise du pétrole a considérablement miné le bilan commercial du pays, et l'obligation de payer les intérêts et de rembourser l'énorme dette nationale pèse lourdement sur l'économie. Une utilisation inadéquate des capitaux provenant d'emprunts contractés en Occident a aussi grevé le budget de l'État. Le manque d'expertise en matière d'investissement efficace et productif n'a pas favorisé non plus l'utilisation optimale des capitaux étrangers. Il faut ajouter à cela un taux d'inflation exhorbitant et la dévaluation de la monnaie pour avoir une vue plus globale de la situation. L'effet conjugué de tous ces facteurs a contribué à une détérioration importante des conditions de vie qui a affecté toute la population, mais en particuler les classes populaires.

Comme l'indique le tableau 1 , le revenu réel par habitant a chuté de près de $25 \%$ de 1970 à 1984 . Cette baisse est encore plus dramatique pour les ouvriers et les employés, dont le niveau de vie a diminué de plus de $36 \%$ entre 1960 et 1984 .

A. Bokor, qui a mené une recherche sur la question de la pauvreté et l'état de privation en Hongrie, note que certains groupes ont été plus touchés par la crise à cause de leur plus grande vulnérabilité et de la faible couverture du système de protection sociale : ce sont principalement les femmes, les familles nombreuses, les personnes âgées et les personnes ayant un faible niveau d'éducation. ${ }^{4}$ 


\section{TABLEAU 1}

\section{Evolution de certains indicateurs des conditions de vie en Hongrie (1950-1984. *)}

\begin{tabular}{|c|c|c|c|}
\hline Période & $\begin{array}{c}\text { Revenu national } \\
\text { par habitant } \\
\text { en valeur réelle }\end{array}$ & $\begin{array}{c}\text { Revenu réel } \\
\text { des ouvriers } \\
\text { et employés }\end{array}$ & $\begin{array}{c}\text { Revenu réel } \\
\text { par habitant }\end{array}$ \\
\hline $1950-1955$ & 125 & 103 & 115 \\
$1955-1960$ & 123 & 147 & 134 \\
$1960-1965$ & 120 & 109 & 117 \\
$1965-1970$ & 136 & 119 & 135 \\
$1970-1975$ & 133 & 118 & 125 \\
$1975-1980$ & 115 & 104 & 109 \\
$1980-1982$ & 105 & 100 & 103 \\
$1982-1984$ & 103 & 94 & 102 \\
\hline
\end{tabular}

* index : La première année de la période $=100,{ }^{3}$ 


\section{Problèmes sociaux}

En Hongrie, plusieurs problèmes sont reliés à la désorganisation familiale. Ainsi, le taux élevé de divorce semble occuper une place prioritaire parmi les problèmes sociaux et ce, dans toutes les strates de la société. Sans être une donnée officielle, le taux de divorce se situe aux environs de $40 \%$, ce qui indique une crise profonde ou tout au moins une mutation importante du système familial.

Les causes de cette crise sont nombreuses. Les uns estiment que la politique économique du pays fait en sorte que la sécurité financière de la famille repose sur l'entrée de deux salaires, ce qui amène les gens à investir beaucoup dans leur travail et moins dans leur famille. D'autres avancent que les changements idéologiques profonds dans la conception même de la famille (rapports hommes-femmes, rapports sexuels, stabilité de la famille, etc.) suivent sensiblement les mêmes transformations qu'en Occident et entraînent donc les couples à remettre plus facilement en question leur situation comme couple et comme famille.

La rupture des couples n'est pas sans provoquer une kyrielle de malaises psychosociaux. Le divorce est souvent associé à des difficultés dans les relations parents-enfants, à une progression des problèmes de comportement chez les jeunes, à des difficultés financières sérieuses, parfois même à la délinquance. Les intervenantes sociales nous ont fait part de leurs préoccupations concernant le climat familial tendu, les relations difficiles entre parents et enfants, et le conflit de générations qui semble s'accentuer constamment. La situation difficile des jeunes et les perspectives d'avenir limitées causent une démotivation et un désabusement.

L'aménagement urbain, et plus spécifiquement l'habitat, font également problème. Une sociologue de l'Office du plan nous a expliqué comment la Hongrie dans son ensemble, et Budapest en particulier, souffrent d'une pénurie chronique de logements, si bien que des couples divorcés sont souvent obligés de partager le même appartement. Même en régime socialiste, on a assisté, au nom de la lutte contre les taudis, à la destruction massive de quartiers ouvriers. Lors d'une visite dans la périphérie nord de Budapest, nous avons pu observer de telles démolitions. Il est vrai que ces maisons anciennes sont remplacées par de grands ensembles où les logements sont plus neufs, mais de l'avis de plusieurs, la qualité de vie dans ces immenses tours demeure plutôt déficiente. De plus, la qualité des constructions est inégale. Souvent situés en périphérie, ces grands ensembles entraînent d'énormes problèmes de transport, et finalement, à cause du manque d'équipements et de facilités, la vie quotidienne crée des situations de tension. 
L'alcoolisme, les rapports interethniques (en particulier entre les populations tzigane et blanche), la délinquance juvénile, le taux élevé de suicide et l'aide aux retraité(e)s, etc., occupent aussi une place importante dans les préoccupations sociales.

L'alcoolisme, par exemple, est considéré comme l'un des problèmes sociaux parmi les plus graves au pays. La consommation est très élevée et, conséquemment, le nombre d'alcooliques progresse sans cesse. Les scènes d'ivresse sont relativement fréquentes, surtout en milieu urbain. Dans ce contexte, on trouve plusieurs centres de désintoxication dans le pays et la police a le devoir d'y amener tout individu trouvé ivre sur la voie publique, de sorte qu'une nuit à l'un de ces endroits amorce souvent une cure prolongée.

Il est important de noter que la Hongrie, avec l'Autriche et la Suisse, détient un des taux de suicide les plus élevés au monde. Par ailleurs, la Hongrie doit faire face, sur le plan intérieur, à certaines tensions sociales et interethniques. Les minorités nationales représentent environ $5 \%$ de la population et sont composées de Tziganes, de Slovaques et de Roumains. Les relations entre ces minorités sont souvent tendues, tant avec les autorités qu'avec la population en général. Finalement on doit signaler l'importante "diaspora hongroise" (environ 2,5 millions selon certaines estimations) qui vit dans les états voisins (Slovaquie et Roumanie) ou dans des pays plus éloignés (Canada et États-Unis).

À l'instar des autres sociétés, la criminalité suit l'évolution sociale générale. Par exemple, durant les années 1948-1952, plusieurs gestes tels que le changement d'emploi, l'abattage d'un porc sans permission ou la tentative de quitter le pays clandestinement étaient considérés comme des crimes entraînant une condamnation. De nos jours, l'ivresse au volant, la possession, l'achat ou la vente de devises étrangères constituent les actes criminels en progression. Ainsi, la manifestation de la criminalité n'est pas statique; elle suit l'évolution de la société hongroise. D'après les estimations, le nombre d'actes criminels est cependant demeuré relativement stationnaire.

En matière de criminalité, la politique est liée à deux contraintes. D'abord, l'attitude punitive de la population rend difficile l'application d'une justice plus humaine, moins axée sur le châtiment. La deuxième contrainte est d'ordre matériel : les prisons sont désuètes et surpeuplées. Bâtir des prisons neuves, modernes ? Une société qui parvient difficilement à loger décemment ses jeunes couples peut-elle se permettre de bâtir des prisons plutôt que des logements? 


\section{Causes des problèmes sociaux}

L'analyse des causes des problèmes sociaux entraîne des opinions divergentes. Selon notre perspective occidentale, la tendance serait de blâmer l'incurie de l'appareil bureaucratique socialiste, mais ce serait trop simpliste. En réalité, plusieurs facteurs explicatifs s'apparentent étrangement, sur certains points, à notre propre conjoncture socioéconomique. Ainsi, l'urbanisation et l'industrialisation accélérées des quarante dernières années ont généré plusieurs situations nouvelles, telle la désintégration du tissu communautaire traditionnel. De plus, un certain immobilisme du gouvernement en matière de prévention des problèmes et de planification des services sociaux, représente un facteur causal qu'il convient également d'identifier.

Les limites dans les échanges commerciaux avec l'Occident, en raison du boycottage permanent des monnaies de l'Est, ont pour effet de ralentir le développement économique. Qu'il suffise de mentionner le développement socio-économique qui n'a pas suivi les aspirations populaires, les changements idéologiques profonds dans la conception de la famille et des rapports hommes-femmes, l'omniprésence de l'État qui a peu à peu démobilisé plusieurs secteurs de la société, de telle manière qu'un nouveau type de dépendance s'est implanté, constituant un important frein au changement.

Dans cette perspective, l'État, qui jouissait d'une certaine popularité, a commencé à être constesté. Un manque de confiance vis-à-vis les dirigeants politiques a été alimenté par l'émergence et surtout par l'étalage de la richesse d'une nouvelle classe qui s'est attribuée certains privilèges. Si la population avait accepté comme inévitable, depuis 1948, la hiérarchie gravitant autour du Parti communiste et les privilèges accordés à ses dirigeants, elle voit maintenant d'un mauvais oil ces nouveaux riches qui se démarquent par l'étalage de leurs biens. Cette nouvelle classe est composée de propriétaires de petites entreprises, de commerçants et d'hommes d'affaires qui, craignant la nationalisation, refusent de réinvestir leurs profits dans leurs entreprises; ils préfèrent se faire bâtir des villas luxueuses sur les collines de la capitale et utiliser leur argent à des fins personnelles. Cette attitude crée un important clivage social et avive parfois l'antagonisme entre la classe laborieuse et les riches propriétaires.

Ces éléments factuels démontrent bien que les problèmes sociaux sont loin d'être réglés à leur source, selon les perspectives déterministes véhiculées par une conception idéaliste du socialisme. La situation économique peu reluisante, la tension naissante entre les nouveaux riches et les travailleurs, la dette énorme du pays et la perte de confiance dans les autorités gouvernementales ont créé une sorte de 
pessimisme généralisé. À part les mesures d'austérité, il est difficile pour le pouvoir en place de proposer des défis stimulants et efficaces, capables de faire sortir le pays de son marasme.

\section{Orientation des politiques sociales}

Dans les pays communistes, la marche vers l'égalité passe par la transformation du système économique. L'abolition de la propriété privée et la centralisation du contrôle des moyens de production constituent un préalable à la réduction des inégalités sociales et économiques. Dans ce contexte, les politiques sociales ont une autonomie toute relative, tenant leurs caractéristiques spécifiques de leur subordination aux politiques économiques. Ainsi, en Hongrie, le système de protection sociale fut traditionnellement organisé autour de l'emploi et de l'entreprise. Les prestations tant en nature qu'en espèces sont liées et conditionnées par le rapport au travail.

Aujourd'hui, il est intéressant de voir le gouvernement critiquer son approche du développement social et chercher de nouvelles avenues de changement. Par exemple, les allocations familiales sont distribuées aux familles avec enfants à la condition expresse qu'un des parents travaille. On ne reconnaît pas la situation de chômage et l'assistance sociale ne correspond qu'à $0.4 \%$ du revenu national. En Hongrie, les dépenses de sécurité sociale représentaient, en 1982, $15 \%$ du P.N.B., comparativement à $26 \%$ en France, $20 \%$ en Italie et $31 \%$ en Suède. Les régimes de maintien du revenu, tout comme la santé et l'éducation, sont relativement développés, contrairement aux politiques d'habitation et aux services sociaux personnels qui restent encore embryonnaires. Par contre, cette situation tend à évoluer rapidement. ${ }^{5}$

En effet, la gravité des problèmes sociaux a fait rejaillir la question des politiques sociales. La résurgence de la pauvreté a provoqué de nouvelles discussions sur la nécessité de réformes politiques et économiques. Une de ces réformes tourne autour de la reconnaissance d'une autorité centrale dans le domaine des politiques sociales : depuis l'abolition du Ministère du bien-être du peuple, en 1950, aucun ministère n'est responsable de la totalité des politiques sociales, dont la gestion est répartie dans plus d'une douzaine de ministères.

Au plan économique, la discussion principale porte sur l'importance à accorder au marché par rapport à l'État et sur la légitimité d'introduire une tarification pour les services publics. En Hongrie comme au Canada, la crise fiscale force l'État à développer des moyens efficaces pour s'assurer des revenus. On parle donc d'introduire l'impôt progressif 
pour les employés et les ouvriers qui, en Hongrie, ne paient pas d'impôt, sinon une contribution pour la sécurité sociale. ${ }^{6}$

Malgré ces limites, quelques exemples de politiques sociales témoignent d'efforts marqués, dans certains secteurs, et permettent d'envisager des solutions à long terme à certains des problèmes sociaux identifiés précédemment. Ainsi, la politique familiale, élaborée depuis 1952, est l'une des plus avant-gardistes au monde. Elle consacre l'égalité des rapports hommes-femmes et vise à supporter la famille dans son cheminement en fonction des changements sociaux et économiques. En ce qui a trait au divorce, la notion de "no-fault divorce" (divorce sans inculpation) est en vigueur et seules les questions de garde des enfants et d'attribution du logement peuvent faire l'objet d'un litige.

Dans le domaine de la sécurité de la vieillesse, on constate une grande considération pour les retraités. Ceux-ci peuvent se retirer graduellement de la vie active, ce qui facilite le passage d'un mode de vie à l'autre. Ils demeurent encadrés par leur syndicat; par ailleurs, voyages, excursions, soirées théâtrales, etc. sont organisés à leur intention. Toutefois, il semble qu'il y ait encore beaucoup de problèmes à solutionner, dont les prestations de retraite peu élevées, les conditions de logement difficiles, etc.

Quant à la santé et à la sécurité au travail, le législateur tente, par divers moyens, d'en améliorer les conditions. Ainsi, bon nombre de manoirs et d'anciens châteaux sont actuellement aménagés en maisons de repos pour les travailleurs. Le programme de vacances-loisirs et l'implication des syndicats dans le domaine de la santé et de la sécurité au travail caractérisent bien la préoccupation du pouvoir politique à cet égard.

Par ailleurs, le système de placement en foyer nourricier expose les jeunes à de fréquents changements de milieu. Pour y remédier, existent les maisons ou villages d'enfants : il s'agit de communautés divisées en familles d'une dizaine d'enfants, chaque famille étant supportée par quelques intervenants sociaux qui y assurent une certaine stabilité. Nous avons pu visiter un de ces établissements, au sud du pays.

Plusieurs autres régimes de sécurité sociale sont intéressants, et les conditions objectives et subjectives semblent réunies pour des changements majeurs. D'une part, les revendications populaires se font de plus en plus pressantes en vue de changements profonds dans le renouvellement et l'application des politiques sociales. D'autre part, en novembre 1987 , et ce pour la première fois depuis 1956, une réunion spéciale du Comité central du P.S.O.H. fut exclusivement consacrée aux questions idéologiques, impliquant bien sûr une réflexion sur la politique sociale. À cette occasion, l'un des responsables de la Section propagande du P.S.O.H. déclarait : "les dirigeants hongrois veulent mener de front la 
revitalisation de l'économie et approfondir la démocratisation du système politique [...] » (L'Humanité, 9 décembre 1987). Aujourd'hui, les dirigeants du pays reconnaissent volontiers que plusieurs réformes se sont " automutilées", notamment en 1968, époque où même le mot "réforme " était banni du vocabulaire en raison des troubles en Tchécoslovaquie. Depuis lors, on a limité les débats à la sphère économique, mais la situation tend à changer radicalement et rapidement.

Dans ce nouveau contexte idéologique, et vu la conjoncture politique interne, les Hongrois souhaitent maintenant des changements en profondeur dans divers secteurs. Dans la population et en particulier dans les milieux intellectuels, une conscience critique des besoins en services sociaux a fait naître des pratiques qu'on peut qualifier "d'alternatives ". Dans les faits, ces pratiques pourront éventuellement être reconnues par un Gouvernement en train d'amorcer sa propre "perestroïka » dans la perspective d'une recherche du mieux-être pour la population. Dans ce même contexte, on parle maintenant du développement possible du service social, ce qui est relativement nouveau en Hongrie.

\section{Condition féminine}

La politique gouvernementale de plein emploi a une signification particulière pour les femmes. Ainsi, $75 \%$ des femmes travaillent, et même $82 \%$, si on considère celles en congé de maternité. ${ }^{7}$ Elles constituent presque $50 \%$ de la population active et, entre 1949 et 1985, leur présence dans le monde du travail s'est accrue dans presque tous les secteurs, catégories et emplois de l'économie hongroise. Le droit au travail salarié leur est maintenant acquis. Leur travail est considéré comme une nécessité dans ce pays où familles et ménages ne sauraient s'en passer. À l'instar de notre société, on retrouve beaucoup de femmes dans des secteurs spécifiques dits "emplois féminins" ou ghettos d'emplois féminins. Même si, contrairement à un pays comme le Canada, plus de femmes occupent des emplois non traditionnels, elles ne gravissent pas également les échelons menant à des promotions et sont victimes de discriminations salariales. On en attribue la cause à une formation inférieure à celle des hommes, et peut-être à une socialisation différente; ce type d'explication n'est toutefois pas évoqué en Hongrie.

Ce taux de participation des femmes à l'économie nationale a nécessité des mesures sociales telles que la préparation professionnelle, la socialisation partielle des enfants à certaines tâches domestiques et la mise en place de services collectifs complémentaires. Ces changements, nécessités par la présence massive des femmes sur le marché du travail, 
sont difficiles et sources de conflit, en raison d'un support social inadéquat, de la persistance des modes traditionnels de relations familiales (i.e. les rôles sociaux) et du peu de prestige accordé à la tâche d'éducation des enfants.

L'implication des femmes, à la fois dans le travail salarié et le travail domestique fait naître des tensions sur les deux plans : inefficacité au travail, fonctions familiales inadéquatement remplies. On parle ici de liens familiaux affaiblis, de problèmes de socialisation, d'attention insuffisante accordée à l'éducation des enfants et de conséquences résultant de la régression du poids démographique de la Hongrie dans l'Europe de l'Est. On parle aussi de fonctions sociales négligées, tels les soins aux personnes âgées, handicapées ou malades, toutes difficultés qu'on qualifie de "difficultés de femmes" à partir d'une notion de responsabilité familiale qui leur est spécifiquement attribuée.

On encourage un meilleur partage des tâches et des responsabilités domestiques entre conjoints. Toutefois, ce sont surtout les couples jeunes, urbains et intellectuels qui vivent ce partage. On estime que la femme passe trois plus de temps que son conjoint, soit trente-cinq heures/semaine, à effectuer le travail domestique en plus du travail salarié. ${ }^{8}$ Les congés de maternité payés sont des initiatives officielles louables, mais servent fréquemment à confier aux femmes, en plus du travail maternel, l'ensemble du travail domestique.

D'autres facteurs contribuent également à une plus grande prise en charge des responsabilités par les femmes; il s'agit du double travail salarié des hommes et, dû aux nombreux divorces, du nombre croissant de familles monoparentales dirigées par des femmes. ${ }^{9}$

Une autre inquiétude causée par la double tâche est le peu de temps consacré à l'éducation des enfants. Ceux-ci reçoivent peu d'attention personnalisée, il y a donc risques d'une carence affective qui pourrait compromettre leur développement harmonieux. Les garderies sont surpeuplées et ne répondent pas adéquatement à la demande.

En définitive, la "question féminine" est intimement liée à la double fonction sur le marché du travail et au foyer. On a tendance à attribuer au travail salarié des femmes ou au fait qu'elles n'assument pas leur double tâche, plusieurs problèmes sociaux: la dénatalité, l'augmentation des divorces, les problèmes d'éducation et de socialisation des enfants. Les femmes deviennent ainsi facilement des boucs émissaires. Cependant, si on situe le problème dans une perspective plus large, on doit constater qu'hommes et femmes s'investissent beaucoup dans leur travail respectif et que la qualité de la vie familiale s'en ressent grandement. Arrondir le budget est une telle tâche qu'on y sacrifie parfois sa santé, son épanouissement personnel et le bien-être familial pour atteindre des conditions matérielles minimales. 
Certaines mesures pourraient pallier aux difficultés mentionnées ; il faudrait rehausser le prestige des activités effectuées au sein de la famille et, plus particulièrement, celles touchant l'éducation des enfants. Ces mesures devraient s'inscrire dans un programme de support à la famille avec enfants, dont le but serait de diminuer les tensions actuelles liées à la vie familiale et de développer une marge de manœuvre plus grande pour les hommes et les femmes dans la sphère du travail salarié et domestique, selon les différentes périodes de leur vie.

Les autorités gouvernementales doivent aussi viser à une intégration de leurs politiques familiales et sociales en matière de population, d'emploi, d'éducation, en ayant comme priorités l'utilisation efficace et la reproduction de la force de travail. Seules la mise en place des conditions d'un véritable choix, et la modulation entre travail salarié, formes flexibles de travail salarié et travail domestique rendront possible l'adéquation entre travail salarié, besoins des familles et besoins de l'économie, où hommes et femmes ne seront plus victimes de leur actuelle double et parfois triple tâche.

Finalement, on souhaite un système plus humain, capable d'équilibrer les valeurs associées au travail salarié et traditionnellement identifiées comme masculines, et les valeurs associées au travail domestique, identifiées comme féminines. Seule une égalité hommes-femmes peut faciliter une véritable émancipation permettant le développement de personnes humaines à part entière.

\section{Perspectives futures : une conclusion}

Après ce périple à la fois modeste et imposant, au terme de ce compte rendu sommaire, quelques brèves réflexions s'imposent afin de dégager le sens de pareille aventure et de repérer les perspectives d'avenir, s'il en est.

Comment ne pas faire état, d'abord, de la rapidité et de l'intensité avec lesquelles s'est établi le contact, la communication entre les deux groupes, et ce, tant au cours des exposés, échanges et discussions parrainés par l'Académie des sciences à Budapest, qu'à l'occasion des visites dans la capitale et au sud du pays.

Que dire des perspectives d'avenir, sinon la poursuite souhaitée, de part et d'autre, de relations d'échange plus ou moins structurées, encadrées et formalisées, échanges fort pertinents et utiles pour les parties en cause. Sur ce plan, à n'en pas douter, l'intérêt existe et est partagé. Les conditions de base, quant à elles, sont de toute évidence déjà en place. En contrepartie, durant l'année qui a suivi notre séjour en Hongrie, les contacts ont été plutôt modestes et peu nombreux, il faut 
bien l'avouer: quelques échanges de lettres et certains envois documentaires de notre part. Toutefois, l'intention de poursuivre, d'aller plus loin même, persiste au sein de notre groupe. Notamment, la production de ce texte en témoigne.

Or, pour nos homologues hongrois, dont l'engagement profond ne fait pas de doute à nos yeux et ouvre de larges perspectives sociales, économiques, culturelles et politiques, la conjoncture actuelle invite à investir provisoirement dans d'autres priorités. Car c'est tout le système et l'organisation sociale du pays qui sont présentement remis en question et qui les interpellent au tout premier chef. La "perestroïka" et la "glasnost" se préparent résolument en Hongrie, et ce depuis fort longtemps, bien avant que la Russie de Gorbatchev n'ait popularisé ces expressions.

En effet, des changements structurels d'une grande importance sont en cours dans ce petit pays de la résistance antisoviétique séculaire. La révolte populaire de 1956 nous le rappelle et la "Relève de la vieille garde politique du pays ", effectuée à la fin de mai 1988, en fournit un témoignage d'une importance stratégique, ${ }^{10}$ dans le contexte plus large de l'ère Gorbatchev et de la "libéralisation » actuelle des rapports Est-Ouest.

Or, ces changements sociaux sont déterminants pour l'existence et le développement, en Hongrie, d'une forme de service social qui nourrisse quelque parenté avec nous. En conséquence, il se peut fort bien que le point d'ancrage des jonctions éventuelles entre les intervenants sociaux de là-bas et ceux d'ici se situe au cœur même du présent "Virage hongrois". 11

Entre-temps, il faut accepter, de part et d'autre, que nos liens soient moins intenses, plus ténus, l'attention devant être braquée provisoirement sur des cibles plus fondamentales. Mais il est d'autant plus pertinent de nourrir ces contacts qu'ils préparent, en termes de possibilités réalistes et souhaitables, le moment où les perspectives d'échange et de coopération pourront prendre des formes plus organisées, intensives et assidues.

C'est sur ce terrain qu'aura lieu, croyons-nous, notre prochain "Rendez-vous ": celui de deux mondes à la recherche de convergences, d'apports nouveaux et de contacts chaleureux, sensibles, respectueux et solidaires.

\section{Notes et références}

* Justin Lévesque a coordonné la rédaction de cet article. Il est professeur à l'École de service social de l'Université de Montréal, ainsi que Lionel Groulx, 
Gisèle Legault et Robert Mayer. Denis Fortin est professeur à l'École de service social de l'Université Laval, André Jacob est professeur au Département de travail social de l'Université du Québec à Montréal et Étienne Vintze est travailleur social au Ministère de la justice du Québec.

** Ce projet a bénéficié de l'appui financier du Ministère des relations internationales du Québec, de l'Université de Montréal et de l'École de service social de l'Université Laval.

1 Voir les trois textes suivants, tirés du même ouvrage: M. LAfOREST, M. NALTCHAYAN et G. BILODEAU, "Une expérience de coopération entre l'École de service social de l'Université Laval de Québec et l'École de formation sociale de l'Université Saint-Joseph de Beyrouth, Liban (pp. 66-86) ; D. FORTIN, A. JACOB et R. MAYER, "Misères et grandeurs du service social international: le cas de la Tunisie" (pp. 123-149); et J.P. DesLAURIERS et al., "Expérience d'échange interuniversitaire France-Québec : similitudes et divergences des pratiques " (pp. 23-41). Dans : D. KIMBERLEY (dir.) Au-delà des frontières nationales - Participation canadienne au travail social et au bien-être social international. Ottawa, Association canadienne des écoles de service social, 1984, 247p.

2 C. Guzzetta, "Social Work in Eastern Europe", International Social Work, vol. 29, no 1, 1986, p. 13.

3 V. Moussatov, "Hongrie: à un tournant", Temps nouveaux, $n^{\circ} 20,1988$, p. 25.

4 A. BOKOR, "Deprivation: Dimensions and Indices", Stratification and Inequality, dans: R. ANDORKAN et T. Kolosı (eds), Hungarian Sociological Studies, no 1, 1984.

5 R. Angelusz, et al., "Public Opinion on Social Policy Issues", dans : Z. FerGe, Policy and Inequality in Hungary, miméo, (1983), p. 6.

6 Z. FERGE, "The Crisis and the "Welfare State" in Eastern Europe with a Focus on Hungary", European Economic Review, vol. 31, 1987 : 212-219.

7 K. KonCz, "Quelques statistiques sur la famille», document non publié, Budapest, Université Karl Marx, 1985.

8 K. KonCZ, "Interdependance Between Women and Men", Third International Working Seminar, Salzburg, 1987.

9 R. DANDURAND, Famille, monoparentalité et responsabilité maternelle, Montréal, thèse de doctorat en anthropologie, Université de Montréal, 1983.

10 P.-A. ComeaU, "Relève de la garde en Hongrie », Le Devoir, 25 mai 1988, p. 8.

11 "Des espaces de liberté en Hongrie" et "Les exclus du parti persistent et signent ", "Le Devoir », 17 mai 1988, p. 5. 